\title{
ABORDAGEM TERAPÊUTICA DE FRATURA RADICULAR COM 30 MESES DE ACOMPANHAMENTO: RELATO DE CASO CLÍNICO
}

\author{
THERAPEUTIC APPROACH ROOT FRACTURE WITH 30 MONTHS FOLLOW: CLINICAL CASE REPORT
}

\author{
Brenda Cabral Sarmento TEIXEIRA ${ }^{1}$; Joana Dourado Martins CERQUEIRA; Sandra de Cassia Santana SARDINHA3; \\ Érica dos Santos CARVALHO 4 \\ 1 - Estudante de graduação do curso de Odontologia, Universidade Federal da Bahia, Salvador, Bahia, Brasil. \\ 2 - Mestre, Professor do curso de Odontologia da Unidade de Ensino Superior de Feira de Santana, Feira de Santana, Bahia, Brasil. \\ 3 - Doutora, Professor Associado I do curso de Odontologia da Universidade Federal da Bahia, Salvador, Bahia, Brasil. \\ 4 - Doutora, Professor Titular do curso de Odontologia da Universidade Federal da Bahia, Salvador, Bahia, Brasil.
}

\section{RESUMO}

Introdução: os traumatismos dentários são muito frequentes e estão entre os principais problemas de saúde pública. As fraturas radiculares tem uma ocorrência que varia entre 0,5 a $7 \%$ e acometem principalmente os incisivos superiores de crianças e adultos jovens. Portanto, necessitam de um diagnóstico preciso para que seja realizado um plano de tratamento e acompanhamento adequado. Objetivo: O presente estudo tem como objetivo relatar o caso clínico de fratura radicular em terço médio de um dente anterior, que foi tratado de forma conservadora e acompanhado por 30 meses. Relato de caso: $\mathrm{O}$ paciente gênero masculino, 42 anos, que procurou atendimento odontológico 23 dias após ter sofrido uma agressão física acometendo a face e o incisivo central superior. Ao exame clínico, observou-se uma discreta extrusão do 21 e ao exame radiográfico periapical detectou-se uma fratura horizontal no terço médio da raiz do mesmo dente. Foi realizada uma contenção semirrígida e desgaste incisal do 21. Após 45 dias, a contenção foi removida e o paciente orientado a retornar periodicamente para controle clínico e radiográfico. Após 30 meses de proservação, verifica-se ausência de dor e de mobilidade, resposta positiva aos testes térmicos de sensibilidade pulpar e cicatrização da linha de fratura ao exame radiográfico e tomográfico. Conclusões: Após este período de acompanhamento, pode-se concluir que o tratamento conservador proposto foi efetivo na manutenção do dente traumatizado, uma vez que este apresenta-se vital e desempenhando suas funções fisiológicas e estéticas

Palavras-chave: Fraturas dos dentes; Traumatismos dentários; Endodontia.

\section{INTRODUÇÃO}

Os traumatismos dentários, juntamente com a cárie e o câncer bucal, estão entre os principais problemas de saúde pública em todo o mundo, trazendo com eles uma série de impactos negativos na qualidade de vida dos indivíduos. Esses impactos negativos podem ser estéticos, fisiológicos ou até mesmo psicológicos ${ }^{1}$.

As injúrias dentárias ocorrem com grande frequência em crianças e em adultos jovens e os fatores etiológicos mais comuns do trauma dental são as quedas, colisões, prática de atividades esportivas, acidentes de trânsito e agressões físicas ${ }^{2}$.

As fraturas radiculares ocorrem em aproximadamente 0,5 a $7 \%$ dos casos em dentes permanentes, principalmente nos incisivos centrais, por possuírem uma posição mais proeminente na arcada dental ${ }^{2-5}$.

As fraturas radiculares horizontais caracterizam-se pela ruptura das estruturas rígidas da raiz, que se divide em dois segmentos: o apical e o coronário. Os principais aspectos clínicos a serem avaliados nesses casos incluem o grau de mobilidade ou deslocamento do fragmento coronário, a sensibilidade à percussão e à palpação, a presença de sangramento no sulco gengival e o estágio de formação da raiz ${ }^{1,3,4,6,7}$.
O exame radiográfico convencional periapical pode revelar dificuldades na localização de algumas fraturas devido à inclinação ou ao deslocamento dos fragmentos, sendo necessárias repetidas tomadas radiográficas em diferentes angulações ${ }^{3}$. A Tomografia Computadorizada de Feixe Cônico (TCFC) tem se tornado a modalidade de escolha em casos de fraturas radiculares horizontais, pois, possibilita uma visualização tridimensional da estrutura dental envolvida, podendo ser útil para detectar a presença, localização, extensão, direção e angulação da fratura sem sobreposição de outras estruturas, reduzindo a chance de um diagnóstico equivocado ${ }^{3,8}$.

Em casos de fraturas radiculares horizontais, existem duas opções de tratamento a serem escolhidas: o tratamento conservador, que visa preservar a vitalidade pulpar onde o tecido dental sofre um processo de cicatrização espontânea; e o tratamento endodôntico e/ou cirúrgico, quando não há chances de garantir a vitalidade pulpar do dente traumatizado ${ }^{2,9}$.

O tipo de cicatrização e o período necessário para que esta ocorra dependerá da injúria pulpar e possível invasão bacteriana na linha de fratura. Esse reparo tecidual pode ser: cicatrização por tecido calcificado; cicatrização através da interposição de tecido conjuntivo e osso; somente formação de tecido conjuntivo; ou pela presença de tecido inflamatório crônico ${ }^{3,10,11}$. 
A proservação dos casos de fratura radicular visa à avaliação contínua de todo o processo de reparação tecidual, bem como o surgimento de possíveis alterações mediatas indesejáveis como lesões periapicais, calcificação pulpar e as reabsorções internas ou externas. Dessa forma, após uma abordagem terapêutica, torna-se essencial manter os pacientes em acompanhamento clínico e radiográfico por um período médio de 05 anos $^{6,7}$.

Apesar da facilidade de acesso às informações e aos meios de comunicação, muitos profissionais da área de saúde, incluindo os Cirurgiões-Dentistas, ainda não são totalmente capacitados para a realização de um primeiro atendimento adequado, frente a situações de traumatismos. Com isso, há uma enorme ocorrência de diagnósticos e planos de tratamentos equivocados, que podem levar ao comprometimento do dente traumatizado ${ }^{12}$.

Dessa forma, o presente estudo visa relatar o caso clínico de um paciente adulto que compareceu ao ambulatório de Endodontia da Faculdade de Odontologia da Universidade Federal da Bahia (FOUFBA) apresentando uma fratura radicular em terço médio.

\section{RELATO DO CASO}

Paciente do gênero masculino, 42 anos, faioderma, sofreu uma agressão física com acometimento da face e envolvimento da região anterior da maxila e recorreu a uma clínica de atendimento de urgência (particular). Após uma radiografia periapical, o dentista plantonista suspeitou de fratura radicular no dente 21 e encaminhou para um cirurgião bucomaxilofacial, que sugeriu a exodontia com posterior colocação de implante.

$\mathrm{O}$ paciente permaneceu sem qualquer tipo de tratamento ou acompanhamento por um período de 23 dias, até procurar o ambulatório de Endodontia da Faculdade de Odontologia da UFBA (FOUFBA), quando foi examinado criteriosamente e um novo exame radiográfico foi realizado para avaliar a evolução do trauma (Figura 1).

$\mathrm{Na}$ anamnese, o paciente relatou ser soropositivo para o vírus da imunodeficiência humana (HIV), mas, estava sob acompanhamento médico. Ao exame clínico, constatou-se ligeira extrusão da unidade 21 e incômodo à palpação e percussão horizontal. Aos testes térmicos de sensibilidade pulpar (Endo Ice ${ }^{\circledR}$ - Maquira Dental, Maringá, Paraná, Brasil), a resposta foi positiva em todas as unidades dentárias envolvidas (11, 12, 21 e 22).

Após a avaliação do paciente, a equipe optou por realizar uma contenção semirrígida com uso do fio ortodôntico 0,5mm (Morelli, Sorocaba, São Paulo, Brasil) e resina composta (TPH Spectrum, Dentsply/ Detrey, Konstanz, Germany) envolvendo as unidades 11, 21 e 22 associados a um ajuste oclusal da unidade 21 (Figura 1).

Após 45 dias com a contenção, o paciente retornou ao ambulatório da FOUFBA para reavaliação das unidades traumatizadas. Foram realizados testes de sensibilidade pulpar que responderam positivamente nas unidades 11, 12, 21 e 22, com ausência de dor e mobilidade. Foi então realizada a remoção da contenção.

O paciente foi orientado a retornar periodicamente para reavaliação clínica e radiográfica, com a finalidade de acompanhar a resposta pulpar e periodontal do tratamento realizado. Sendo assim, nas reavaliações de 3 meses; 5 meses, 20 meses e 25 meses após o trauma (Figura 2), o paciente apresentou respostas positivas aos testes de sensibilidade pulpar nas unidades 11,12, 21 e 22, ausência de dor e mobilidade e indícios radiográficos de cicatrização/consolidação da fratura radicular
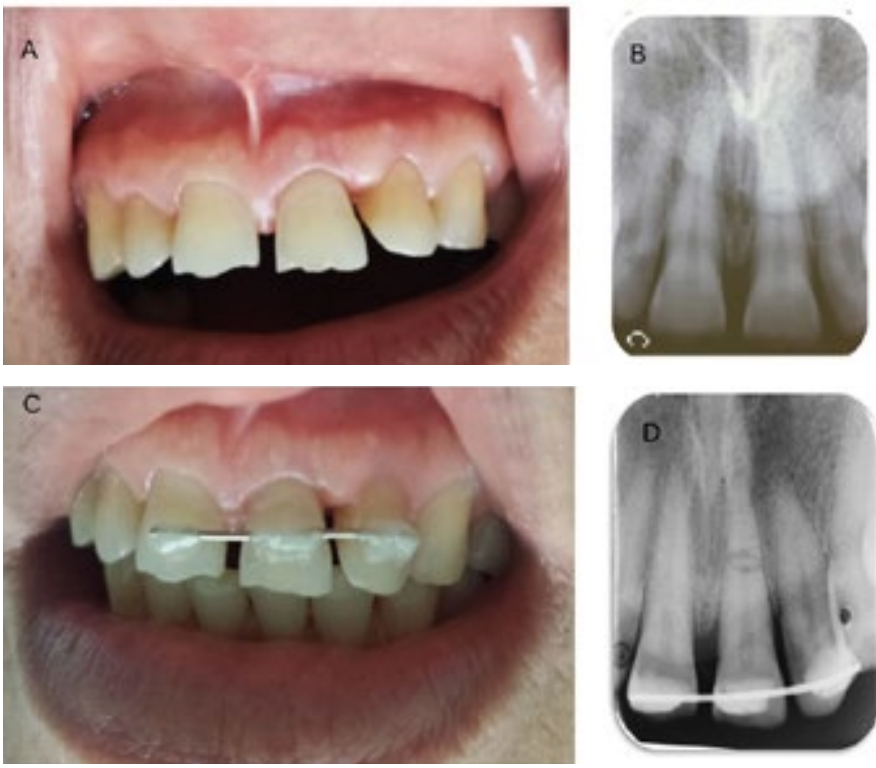

Figura 1 - A) Avaliação clínica inicial do paciente. B) Avaliação radiográfica inicial C) e D) Avaliação clínica e radiográfica após a realização da contenção semirrígida e desgaste incisal da unidade 21.
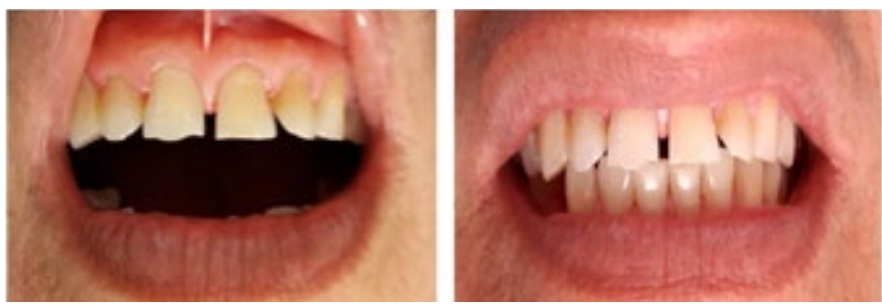

Figura 2 - A) Imagem radiográfica 03 meses após o trauma. B) Imagem radiográfica 05 meses após o trauma. C) Imagem radiográfica 20 meses após o trauma. D) Imagem radiográfica 25 meses após o trauma.

Trinta meses após o trauma, foi solicitada uma Tomografia Computadorizada de Feixe Cônico para melhor observação da posição dos fragmentos e do processo de cicatrização. Os achados tomográficos demonstraram nitidamente a interposição dos fragmentos e a cicatrização do tecido ósseo, além da ausência de sinais de reabsorções internas ou externas (Figura 3). A avaliação clínica mostrou integridade da unidade dentária, sem mobilidade ou escurecimento dental (Figura 4).

\section{DISCUSSÃO}

As fraturas radiculares horizontais pode ser uma consequência dos traumatismos dentários e o cirurgião-dentista deve estar apto para realizar o diagnóstico e tratamento mais eficaz para a manutenção do dente envolvido ${ }^{2-5,11}$. Essa injúria traumática acomete mais frequentemente os incisivos superiores e podem ocorrer em qualquer ponto da raiz, porém, são mais comuns no terço médio ${ }^{3,4,7}$. O prognóstico das fraturas do terço médio é mais favorável, chegando a $78 \%$ de possibilidade de manutenção da vitalidade pulpar, quando estes dentes são adequadamente acompanhados ${ }^{2}$. Isto foi observado no caso clínico descrito, onde a localização da fratura no terço médio radicular, com a justaposição dos fragmentos e ausência de contaminação, favoreceram o prognóstico ${ }^{3,13}$.

O diagnóstico deve ser baseado na associação do exame clínico com o radiográfico e o protocolo de tratamento consiste em 
reposicionar o fragmento coronário, checando a sua posição radiograficamente e estabilizá-lo com uma contenção semirrígida durante 04 semanas para induzir a cicatrização dos fragmentos ${ }^{6}$. Recomenda-se monitorar a vitalidade pulpar e, caso ocorra a necrose, está indicado o tratamento endodôntico do fragmento coronário até a linha de fratura, com trocas de medicação intracanal ${ }^{3,7,10}$.

O acompanhamento clínico e radiográfico dos dentes traumatizados deve ser mantido, pois, a interrupção da transmissão dos estímulos nervosos provocada pela fratura radicular pode gerar respostas falso negativas até quase 1 ano após o trauma, e assim levar um profissional inexperiente a conduzir erroneamente o tratamento, supondo que há uma condição de necrose pulpar ${ }^{14}$.

A contenção é um recurso auxiliar que visa a justaposição dos fragmentos, mantendo as unidades em posição de repouso, a fim de ajudar no reparo do feixe vásculo-nervoso e das fibras periodontais. O tipo de contenção varia de acordo com a complexidade do caso, podendo ser Flexíveis, com fios de Nylon (n⿳ำ 70 a 80) quando há apenas lesão nos tecidos de sustentação, ou Semirrígidas, com fio ortodôntico $(0,2$ a $0,5 \mathrm{~mm})$, utilizada em casos onde há maior grau de mobilidade e acometimento da unidade, como nas fraturas radiculares ${ }^{7,15}$. Se a fratura for localizada no terço cervical, recomenda-se uma contenção por um maior período, até 4 semanas ${ }^{6,12,16}$.

Estudos mostram que ainda existe um despreparo e dúvida por parte de muitos cirurgiões-dentistas diante de um tratamento de urgência de um dente traumatizado ${ }^{11,17}$. No caso em questão, o paciente passou por 2 cirurgiões-dentistas que não souberam como intervir corretamente, porém, mesmo após alguns dias após o trauma, o atendimento na FOUFBA seguiu este protocolo atual de contenção e proservação, visando a cicatrização da linha de fratura.

O tipo de cicatrização depende da eventual injúria à polpa e possível invasão bacteriana na linha de fratura. Estudos afirmam a existência de quatro principais tipos de reparo tecidual: o reparo pela união dos fragmentos através da cicatrização por tecido calcificado; cicatrização através da interposição de tecido conjuntivo e osso entre os fragmentos; formação de tecido conjuntivo ou ocorrência de uma "falsa união", pela presença de tecido inflamatório crônico entre os fragmentos, não havendo a cicatrização e estabelecendo o pior prognóstico ${ }^{5,10}$.

Segundo a literatura, a reação tecidual mais frequente na linha de fratura é a interposição do tecido conjuntivo, que ocorre em quase $65 \%$ dos $\operatorname{casos}^{5}$. No entanto, pode-se considerar que o melhor tipo de cicatrização é aquela que possibilite a manutenção da vitalidade pulpar ${ }^{12}$. No caso descrito, observou-se através da análise das imagens radiográficas, uma considerável interposição de tecido conjuntivo fibro-ósseo, que garantiu uma integridade da lâmina dura e da cortical vestibular daquela unidade.

Os dentes permanentes jovens possuem maior chance de recuperação diante de um trauma quando comparado a um dente adulto, completamente formado, uma vez que possuem uma maior vascularização e um maior número de células ${ }^{3,9,12}$. No caso em questão, contudo, apesar da idade do paciente não ser considerada ideal para respostas positivas e o paciente ser portador do vírus HIV (possível redução da resposta imunológica) ${ }^{17}$, a unidade envolvida permaneceu vital durante todo o período de acompanhamento. Isto pode ser explicado pela correta reposição dos fragmentos, durante o período preconizado pela literatura, evitando a contaminação da região fraturada.
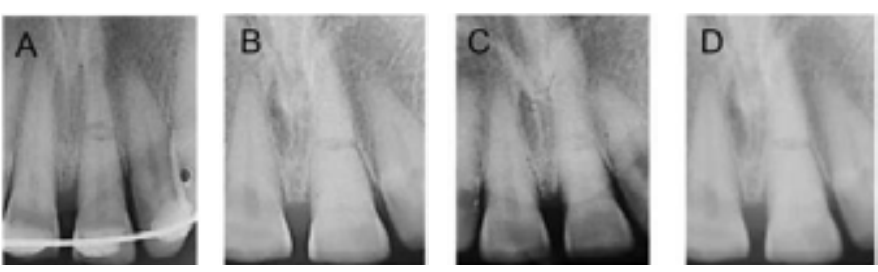

Figura 3 - Cortes de TCFC 30 meses após o trauma. A) Reconstrução tridimensional. B) Cortes sargitais indicando união dos fragmentos da raiz com interposição de tecido fibro-ósseo. C) Cortes Axiais indicando ausência de reabsorções internas e ou externas.
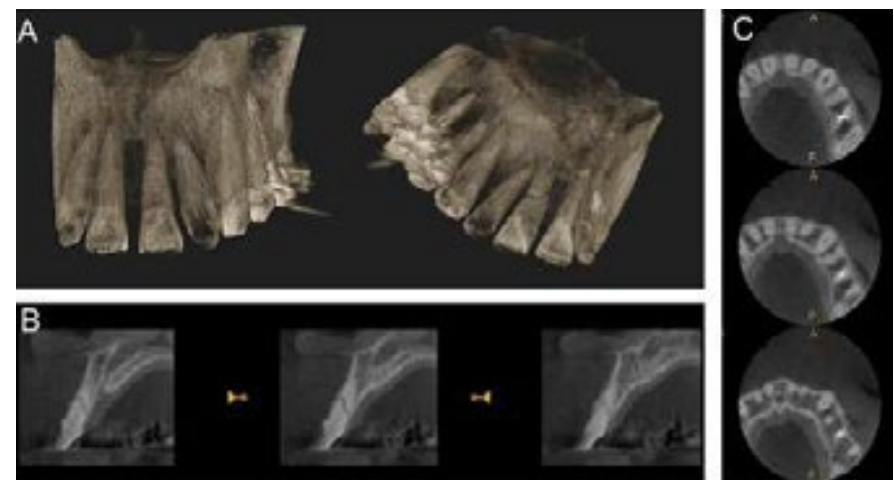

Figura 4 - Imagem clínica do paciente, 30 meses após o trauma.

As mudanças nas técnicas favoreceram a abordagem conservadora das fraturas radiculares horizontais em diferentes níveis, permitindo que o paciente mantenha sua dentição natural com um custo mais acessível, sendo uma alternativa mais viável do que as técnicas mais caras e invasivas ${ }^{4}$. Como neste caso, o paciente apresentou respostas positivas e o tratamento de escolha foi apenas o acompanhamento, através de radiografias e testes de sensibilidade realizados periodicamente. Observa-se, entretanto, uma redução da luz do canal sugestiva de uma resposta degenerativa da polpa ao trauma sofrido. Isso tem sido relatado como uma das sequelas mais frequentes nos casos de fratura radicular, sem, contudo, comprometer a sua manutenção na $\operatorname{arcada}^{2,18}$.

A Tomografia Computadorizada de Feixe cônico (TCFC) têm sido muito utilizada no diagnóstico e acompanhamento dos dentes traumatizados, uma vez que possibilita uma avaliação tridimensional das estruturas envolvidas ${ }^{8-10,19}$. Assim como outros estudos, neste caso foi possível observar por meio dos cortes sagitais que a fratura transversa no terço médio da raiz antes observada nas radiografias periapicais, apresentava-se oblíqua na tomografia ${ }^{8}$. Além disso, após o período de acompanhamento, notou-se ausência de reabsorções radiculares e presença de integridade da lâmina dura e da cortical óssea vestibular, o que prova a precisão desses exames tridimensionais.

De acordo com a literatura avaliada e o caso descrito, pode-se inferir que o tratamento correto e imediato, associado ao acompanhamento clínico, radiográfico e tomográfico poderão favorecer o reparo e manutenção de dentes com fratura radicular sem maiores sequelas que possam complicar o tratamento ou até mesmo levar a perda do dente. Para tanto, os profissionais de saúde, especialmente os cirurgiões-dentistas devem estar capacitados e cientes da importância de um tratamento conservador adequado nesses casos. 


\section{CONSIDERAÇÕES FINAIS}

O tratamento conservador da fratura radicular pode apresentar bom prognóstico, como foi demonstrado no presente caso clínico. Após 30 meses de acompanhamento clínico, radiográfico e tomográfico, observou-se ausência de lesão periapical e reabsorções, resposta positiva aos testes de sensibilidade pulpar e cicatrização por tecido conjuntivo fibro-ósseo entre os fragmentos. Para tanto, o profissional deve realizar um bom diagnóstico e adequado tratamento inicial.

A utilização da Tomografia computadorizada de feixe cônico faz-se extremamente importante no diagnóstico e acompanhamento dos casos de fraturas radiculares.

\section{REFERÊNCIAS}

01. PetersenPE,BourgeoisD, OgawaH,EstupinanDS,NdiayeC.Theglobal burdenoforaldiseasesand riskstooralhealth. BullWorldHealthOrgan. 2005; 83(9): 661-669.

02. Andreasen JO, Andreasen FM, Andersson L (eds.). Textbook and Color Atlas of Traumatic Injuries to the Teeth. 4 edition. Munksgaard: Kopenhagen; 2007. p. 337-71.

03. Monteiro MR, Ferraz RCC, Silva EJNL, Almeida JFA. Diagnosis of a horizontal root fracture during retreatment of a maxillary canine utilizing an electronic apex locator and monitoring with CBCT: a case report. Braz Dent Sci. 2013; 16(4): 99-104.

04. Sisodia N, Manjunath MK. Conservative Management of Horizontal Root Fracture. J Clin Diagn Res. 2015; 9(8): ZD04-ZD06.

05. Kapoor S, Bansal P, Chandran S, Agrawal V. Surgical Management of a Non-healing intra-alveolar root fracture associated with pulpal calcifcation and root resorption: a case report. J Clin Diagn Res. 2015; 9(6): ZD03-ZD05.

06. Diangelis AJ, Andreasen JO, Ebeleseder KA, Kenny DJ, Trope M, Sigurdsson A, et al. International Association of Dental Traumatology guidelines for the management of traumatic dental injuries: 1 . Fractures and luxations of permanent teeth. 2012: 2-12.

07. Erdem AP, Ozdas DO, Dincol E, Aren G. Case Series: Root healing with MTA after horizontal fracture. Eur Arch Paediatr Dent. 2009; 10(2): 110-113.

08. Ronnachat R, Patchanee C. Differences in Healing of a Horizontal Root Fracture as Seen on Conventional Periapical Radiography and Cone-Beam Computed Tomography. Case Rep Den. 5: 2017.

09. Fagundes DS, Mendonça IL, Albuquerque MT, Inojosa IF. Spontaneous healing responses detected by cone-beam computed tomography of horizontal root fractures: a report of two cases. Dent Traumatol. 2014; 30(6): 484-7.
10. Tsukiboshi MO. Optimal use of photography, radiography and micro computed tomography scanning in the management of traumatizes teeth. Endod Topics. 2006; 14(1): 4-19.

11. Gharechahi M. Horizontal root fracture accompanied by luxation of coronal fragment in a maxillary central incisor: a case report. J Dent Res Dent Clin Dent Prospect. 2013; 7(4): 244-247.

12. Frujerimde L, Costa Jr. ED. Effect of a single dental health education on the management of permanent avulsed teeth by different groups of professionals. Dent Traumatol. 2009; 25(3): 262-71.

13. Tomazella CR. Tratamento e prognóstico das fraturas radiculares: revisão de literatura [tese da especialização]. Campinas: Faculdade de Odontologia de Piracicaba; 2015.

14. Liao Q, Ye W, Yue J, Zhao X, Zhang L, Zhang L, Huang D, Zheng Q. Self-repaired Process of a Traumatized Maxillary Central Incisor with Pulp Infarct after Horizontal Root Fracture Monitored by Laser Doppler Flowmetry Combined with Tissue Oxygen Monitor. J Endod. 2017; 43(7): 1218-1222.

15. Losso EM, Tavares MCR, Bertoli FMP, Baratto-Filho F. Dentoalveolar trauma in the primary dentition. RSBO. 2011; 8(1): e1-20.

16. Cvek M, Andreasen JO, Borum MK. Healing of 208 intraalveolar root fractures in patients aged 7-17 years. Dent Traumatol. 2001; 17(2): $53-62$.

17. Oliveira FLD, Santa-Rosa CC, Bambirra BHS, Oliveira EMC, Castilho LS, Silva MES. Root fracture in patient in the pre-liver transplantation phas. RGO. 2017; 65(2): 185-190.

18. Morello J, Ribeiro FC, Roldis A, Pereira RS, Barrosos JM, Intra JBG. After effects following traumatic dental Injury with endodontic involvement. Rev bras pesq saúde. 2011; 13(2): 68-73.

19. Salineiro FCS, Pinheiro LR, Santos Júnior O, Cavalcanti MGP. Detection of horizontal root fracture using four different protocols of cone-beam computed tomography. Braz Oral Res. 2015; 29(1): 1-6.

20. Westphalen VPD, Carneiro E, Fariniuk LF, SIlva-Neto UX, Kowalczuck A. Maintenance of pulp after horizontal root fractures in three maxillary incisors: a thirteen-year evaluation. Iran Endod J. 2017; 12(4): 508-511.

21. Andreasen JO, Ahrensburg SS, Tsilingaridis G. Root fractures: the influence of type of healing and location of fracture on tooth survival rates-an analysis of 492 cases. Dent Traumatol. 2012; 28(5): 404-9.

22. Çiçek E, YJlmaz N, Koçak MM. Intraradicular Splinting with Endodontic Instrument of Horizontal Root Fracture. Case Reports in Dentistry. 2015; 24(5): 578-580.

\section{ABSTRACT}

Introduction: dental injuries are very frequent and are among the main problems of public health. The root fractures occur between 0,5 a $7 \%$ and mainly affect the central incisors of children and young adults. So, its necessary a certain diagnosis and a proper treatment and follow-up plan. Objective: this study aims to report the clinical case of root fracture in the middle third of a previous tooth, which was treated conservatively and followed up for 30 months. Case report: the male patient, 42 years old, who sought dental care 23 days after having suffered a physical aggression involving the face and upper central incisor. Clinical examination revealed a discrete extrusion of the 21 and periapical radiographic examination a horizontal fracture was detected in the middle third of the root of the same tooth. A semi-rigid restraint and incisal wear of the 21 were performed. After 45 days, a restraint was removed and the patient was instructed to return periodically for clinical and radiographic control. During a period of 30 months of proservation, there is no pain and mobility, positive response to the thermal pulpal sensitivity tests and healing of the fracture line in the radiographic and tomography examination. Conclusions: After this follow up, it was concluded 
that the conservative treatment proposed was effective in the maintenance of the traumatized tooth, since it is vital and performs its physiological and aesthetic functions.

Keywords: Tooth fractures; tooth injuries; Endodontics.

\section{AUTOR PARA CORRESPONDÊNCIA}

Joana Dourado Martins Cerqueira

Rua Quintas do Sol, n²0, Condomínio Quintas do Sol Ville

2, casa 14, Bairro Parque Ipê, Feira de Santana, BA.

Tel: (55) (75) 3161-8248

E-mail: martinsjoana_1@hotmail.com 